Chemical Physics 136 (1989) 413-428

North-Holland, Amsterdam

\title{
LINE-NARROWING SPECTROSCOPY IN AMORPHOUS SOLIDS THROUGH POLARIZATION DETECTION OF SPECTRAL HOLES. I. PRINCIPLES
}

\author{
Bernhard DICK \\ Max-Planck-Institut für biophysikalische Chemie, Abteilung Laserphysik, D-3400 Göttingen, FRG
}

Received 11 April 1989

\begin{abstract}
Detection of spectral satellite holes by polarization spectroscopy is proposed as a method to measure the line-narrowed electronic absorption spectrum of a large molecule in an amorphous solid at low temperature. The linewidths of the zero-phonon holes yield information about molecular dephasing and the correlation of the inhomogeneous distribution of the two molecular transitions involved in the hole-burning and the detection step. Constructive or destructive interference of the signal light field with an intentionally admixed background light field yields information on the symmetry of vibronic states.
\end{abstract}

\section{Introduction}

The electronic absorption spectra of large molecules contain a wealth of information about the intramolecular relaxation dynamics and the intermolecular interactions. Unfortunately under normal conditions the fine structure of these electronic spectra is obscured due to several broadening mechanisms which cause the absorption spectrum to appear as few broad bands only. It is hence of interest to develop line-narrowing techniques which are capable of suppressing the broadening as far as possible and uncover the underlying individual transitions. The three most important types of broadening are thermal congestion, homogeneous broadening, and inhomogeneous broadening.

Thermal congestion is due to the fact that at finite temperature many states of a molecule can be populated, and the absorption spectra of all these species overlap. Lowering of the temperature will reduce this broadening to the same extent to which the population of the molecules is condensed into a single ground state. In the condensed phase the product states of the ground state of the isolated guest molecule with low-frequency phonon states of the host lattice constitute a dense manifold of low-lying effective ground states for the guest molecule. Very low temperatures are needed to reduce the statistical weight of these phonon-coupled states below that of the zero-phonon state.

The two other sources of broadening - homogeneous and inhomogeneous - are also affected by lowering the temperature. They result from the modulation of the molecular transition frequencies by the fluctuating perturbations exerted upon the guest molecule by the surrounding host molecules. If the autocorrelation time of these fluctuations is much longer than the time required by Heisenberg's uncertainty principle to measure the molecular transition frequency, the resulting broadening is inhomogeneous: each measurement performed on an individual molecule will yield a value of the transition frequency characteristic for its particular environment. In the limit of infinitely slow perturbations the inhomogeneous broadening can be modelled by a static distribution of transition frequencies. On the other hand, when the autocorrelation time of the fluctuations is much shorter than the time that would be needed to measure the spectral shifts between various sites, the transition will be homogeneously broadened.

In real systems fluctuations on all possible timescales exist, and both homogeneous and inhomogeneous broadening is present. However, lowering of the temperature will slow down many of the perturbing processes, e.g. rotational relaxation of the host molecules. Hence many perturbing processes that 
contribute to homogeneous broadening at high temperatures will lead to inhomogeneous broadening at low temperatures. At the limit of zero temperature only spontaneous decay processes should remain as a source for homogeneous line broadening (lifetime broadening), and all other line broadening should be inhomogeneous.

A special case of inhomogeneous broadening encountered in the gas phase is Doppler broadening. Several line-narrowing techniques have been developed to overcome this type of broadening, e.g. saturation spectroscopy [1-7], two-photon absorption of counter-propagating waves [8-11], or molecular beam spectroscopy [12-14]. A widely applied technique for the study of condensed phases is fluorescence line narrowing [15-19]. It can be applied to strongly broadened inhomogeneous systems with amorphous structure like glasses but is more often used for the study of crystalline samples like Shpol'skii matrices, where individual fluorescence lines can be assigned to individual sites. The linewidth of the transition which is resonantly excited can usually not be studied in this way since the fluorescence cannot be sufficiently discriminated against the scattered laser light.

The line-narrowing potential of several coherent nonlinear optical processes like coherent anti-Stokes and Stokes Raman scattering (CARS, CSRS) [2022 ], or sum- and difference-frequency generation (SUM, DIF) [23] has been predicted and in some cases verified [24]. An interesting feature of these processes are resonances associated with transitions between excited states [25-30]. However, so far these techniques have not found wider application for linenarrowing purposes, presumably because of the special nature of the inhomogeneous distribution required (e.g. anticorrelation for CARS and SUM).

A nonlinear but incoherent method applicable to line-narrowing spectroscopy is spectral hole-burning $[31,32]$. This method has been widely applied to the study of resonant holes which yields the homogeneous linewidth of the molecular transition excited with the hole-burning laser. This is usually the vibrationless transition to the first electronically excited singlet state with a homogeneous linewidth of a few $\mathrm{MHz}$ [33-35]. However, hole-burning into transitions to vibronic states [36,37] and higher electronically excited states [38] has also been reported. In several instances vibronic satellite holes have been observed [38-42], but the method has to our knowledge not been used to obtain the complete line-narrowed spectrum of an inhomogeneously broadened electronic transition. One reason for this might be the fact that vibronic satellite holes are usually much weaker than the resonant hole, and a sensitive detection method is required. In this paper we investigate polarization spectroscopy as such a sensitive detection method. This method has been developed originally for gasphase studies [43-45] and has also been applied to liquids [46-51]. Few applications to solid samples at low temperature have been reported [52-54] presumably since birefringence from other sources (e.g. strain) is a serious problem. However, as will be shown in this paper, this birefringence can be compensated to a large extent.

In section 2 we shall describe how vibronic satellite holes can be generated in hole-burning experiments, and discuss the conditions under which this effect can be used for line-narrowing spectroscopy. In section 3 the use of polarization spectroscopy for the detection of spectral holes is explained and a brief theory of the spectral lineshape is presented. The experimental technique is described in section 4 . Some results are presented and discussed in section 5 . The sensitivity of polarization spectroscopy and ordinary transmission spectroscopy are compared for the detection of resonant and satellite holes in a sample of meso-tetraphenylporphin (TPP) in polymethylmethacrylate (PMMA) at $10 \mathrm{~K}$. The photochemical reaction of this compound leading to spectral hole-burning is the tautomerization of the inner hydrogen atoms shown in fig. 1. Finally it is shown that the interference of the signal wave with a background wave (produced

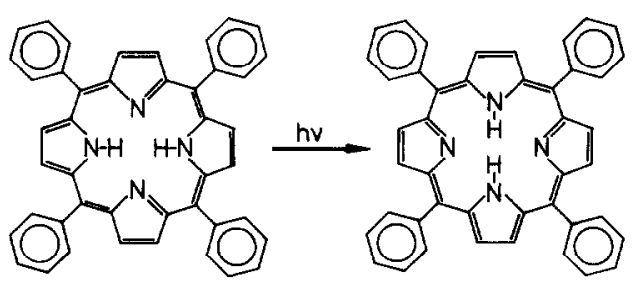

Fig. 1. Structural formula of meso-tetraphenylporphin (TPP) and its photochemical reaction leading to spectral hole-burning. The tautomerization of the inner hydrogen atoms is equivalent to a rotation of the molecule by $\pi / 2$ about the symmetry axis perpendicular to the molecular plane. 
through deliberately detuning the polarizer from perfect crossing with the analyzer) will yield information on the relative orientation of transition dipole moments. This can be used to assign the symmetry of the vibronic states. These results allow the conclusion that hole-burning of satellite holes combined with the detection method of polarization spectroscopy is a very powerful tool for line-narrowing spectroscopy.

\section{The origin of spectral satellite holes}

Spectral hole-burning is a method to obtain the homogeneous linewidth from the inhomogeneously broadened spectrum. A narrow-bandwidth laser excites only a small subensemble of the molecules, whose resonance frequency is within the homogeneous linewidth of the laser frequency. As long as these molecules have not returned to the ground state, the absorption spectrum of the sample taken with a second light source will display a spectral hole: the absorption spectrum of the laser-excited molecules is now missing. When the laser-excited molecules undergo a photochemical reaction, the process is called photochemical hole-burning, and the spectral holes are permanent as long as no backreaction occurs [55-58]. Permanent spectral holes can also be effected by photophysical processes which modify the structure of the environment of a guest molecule and thus shift its transition frequency to another position within the inhomogeneous band [59-61]. Finally, spectral holes can be transient when the molecules are pumped to a metastable bottleneck state $[38,62-64]$.

When the hole-burning process removes a homogeneous subensemble of molecules from the inhomogeneous ensemble, the whole absorption spectrum of these molecules is removed from the absorption spectrum of the sample. This can lead to the occurrence of satellite holes, as schematically shown in fig. 2. A necessary condition for the occurrence of sharp satellite holes is that the inhomogeneous distribution of the transition excited by the hole-burning laser and the transition in which the satellite hole is observed are correlated. Full correlation means that each molecule has the homogeneous absorption lines of both transitions at the same relative position within the two inhomogeneous absorption bands. With other words, the difference of the

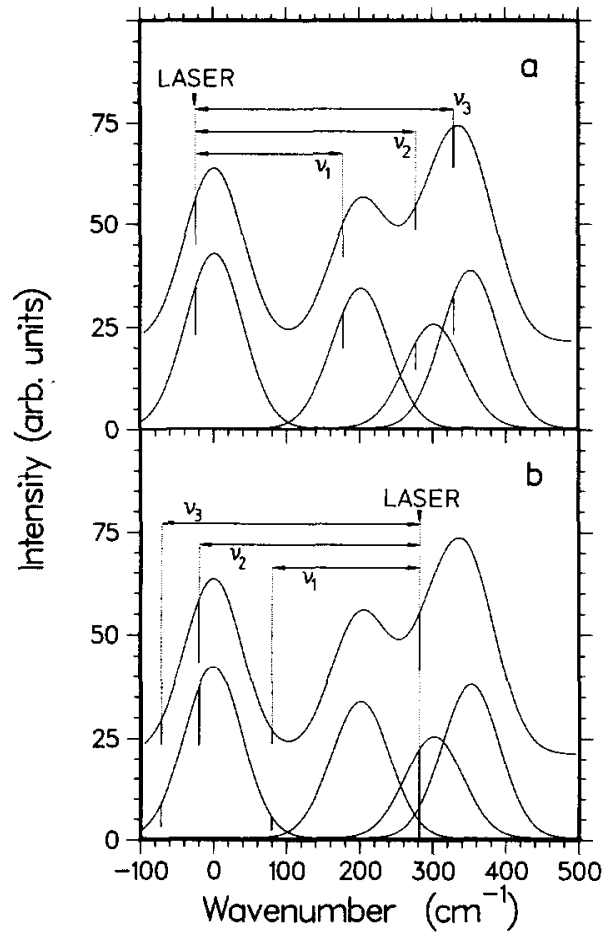

Fig. 2. Schematic representation of satellite hole production. (a) (type-I) hole-burning into the electronic origin band produces a single hole in the inhomogeneous ensemble with a satellite hole for each vibration. (b) (type-II) hole-burning into the region of overlapping vibronic bands produces several spectral holes in the inhomogeneous distribution of the electronic origin band, one for each vibration.

two transition frequencies should not be inhomogeneously distributed but should be equal for all molecules in the ensemble. Such a situation can be expected to occur for the vibronic transitions belonging to the same electronic transition: The interaction of the guest molecule with the surrounding host material will mainly affect the outer part of its electronic wavefunction. This will alter the energies of the electronic states and hence shift all electronic transition frequencies. The equilibrium position of the nuclei and the curvature of the electronic potential curves along the coordinates of nuclear motion should be much less affected. Therefore, only a small effect on the vibrational frequencies within the same electronic state is expected, and in the ideal case of complete correlation all molecules have the same vibrational frequencies. Hence the inhomogeneous 
broadening can be viewed as only affecting the energetic separation of the two electronic hypersurfaces without affecting their shapes. Such a view is supported by the observation that electronic transition frequencies are often strongly shifted in going from the vapor phase to solvents of different polarity or polarizability, while the vibrational spacings within the same electronic state remain almost constant.

In fig. 2 it is schematically shown how hole-burning can be used to obtain a line-narrowed spectrum of the vibronic structure of an electronic transition with fully correlated inhomogeneous distributions for all vibronic transitions. An absorption spectrum of four bands has been simulated, the first corresponding to the electronic origin, and three overlapping bands corresponding to vibronic transitions with vibrational wavenumbers $\nu_{1}, \nu_{2}$, and $\nu_{3}$. In both parts of fig. 2 the four Gaussian bands of the four inhomogeneously broadened transitions are shown below the sum of these bands representing the absorption spectrum. It is obvious that inspection of the absorption spectrum alone would not reveal the transition with wavenumber $\nu_{2}$, and would yield too small a value for the vibrational wavenumber $\nu_{3}$. When a hole is burned into the electronic origin band, three sharp satellites will appear, whose wavenumber shifts with respect to the hole-burning laser wavenumber will permit an accurate measurement of the three vibrational wavenumbers, as shown schematically in the upper part of fig. 2 . The reversed situation is shown in the lower part of fig. 2. When the laser irradiates the sample in the spectral region of the three overlapping vibronic bands, three homogeneous subensembles of the total inhomogeneous ensemble are excited, one for each vibronic band in resonance with the laser. Hence three sets of spectral holes are produced. The lowest-energy hole of each set corresponds to the 00 transition and can be observed by scanning the absorption spectrum of the electronic origin band. The wavenumber differences between these holes and the hole-burning laser wavenumber will yield the vibrational wavenumbers. This second method might seem indirect, and the reader will notice that the three holes in the inhomogeneous distribution will also appear in each of the vibronic bands, which could make the high-energy part of the spectrum difficult to interpret. (However, only a scan over the electronic origin band is needed to find all holes produced in the inhomogeneous distribution, and thus to find all vibrational wavenumbers.) Furthermore, in this method many experiments have to be performed with different settings of the wavenumber of the hole-burning laser, since each experiment probes only a spectral range of the size of the inhomogeneous width.

However, the indirect method offers several important advantages over the direct method when the Franck-Condon factors of the vibronic transitions are smaller than that of the electronic origin, or when the homogeneous width of the electronic origin is much narrower than those of the vibronic transitions. The reason for this is the largely differing amount of molecules that can be burned in both methods before saturation sets in.

In the direct (type-I) method, shown in fig. 2a, saturation will set in when about $10 \%$ of the molecules having their zero-phonon line at the laser wavenumber have been burned. The fraction of molecules burned is given by the ratio of the area under the resonant hole to the total area of the inhomogeneous absorption band. For long-lived electronic states and narrow-bandwidth laser excitation this ratio is usually below $10^{-5}$. The area under the satellite holes is given by that of the resonant hole times the ratio of the corresponding Franck-Condon factors. Hence, when the Franck-Condon factor of the vibronic transition is much smaller than that of the electronic origin, or when the homogeneous width of the vibronic transition is larger than that of the electronic origin (or the width of the laser), the peak change in optical density at the spectral position of the satellite hole will be much smaller than at the resonant hole. In this case the detection of direct satellite holes will encounter serious signal-to-noise problems.

These disadvantages of the direct method turn out to be advantages for the indirect (type-II) method shown in fig. 2b. Even if the optical density of the sample at the wavenumber of hole-burning is small, burning of a certain fraction of molecules at this wavenumber will remove a corresponding fraction of absorption from the electronic origin band. Hence the hole will be amplified by the ratio of the FranckCondon factors of the 00 transition to that of the vibronic transitions. Further, several holes are produced simultaneously, each of which can be as deep as $10 \%$ before saturation sets in. The width of these 
holes observed in the electronic origin band is the homogeneous width of the corresponding vibronic transition (in the model of perfect correlation of the inhomogeneous distributions). Since these widths are usually much larger than the homogeneous width of the vibrationless transition, the total fraction of molecules burned can be several orders of magnitude larger than in the case of direct (type-I) hole-burning.

\section{Polarization spectroscopy of spectral holes}

A straightforward method to obtain a line-narrowed absorption spectrum is to measure the absorption spectra of the sample prior to and after holeburning. The difference of these absorption spectra is the absorption spectrum of the homogeneous subensembles removed from the initial ensemble through hole-burning. In most cases the spectral hole corresponds to only a very small increase in light transmission. Hence very accurate measurements of the absorption spectra are required to obtain a difference spectrum with modest signal-to-noise ratio. The contrast ratio of a spectral hole, defined as the relative increase in light transmission, can be drastically improved through application of polarization spectroscopy $[53,54,65]$. In this technique the transmission of a probe light beam through the sample placed between crossed polarizers is measured. No transmission occurs for an isotropic sample. Hole-burning with polarized light makes the sample anisotropic at the wavenumbers of the spectral holes, and transmission of probe light will occur at these wavenumbers. In the following a brief theoretical treatment ${ }^{\# 1}$ of this effect will be given in three steps: First, the modification of the spectral and orientational distribution function of the inhomogeneous ensemble through hole-burning is calculated. Then the propagation of the probe beam through this modified sample is considered. Finally, the expected zero-phonon lineshape is calculated for a system with strictly correlated inhomogeneous distribution.

The molecular level system that will be used for the description of photochemical hole-burning is sche-

\#1 A similar theory has been developed in ref. [53] for resonant spectral holes in crystalline systems, in which the transition dipole moments are oriented in a finite number of directions. matically shown in fig. 3. The molecules have two stable states: the ground state $|a\rangle$ of the educt, and the ground state $|r\rangle$ of the product. Hole-burning is performed with a light beam of frequency $\omega_{\mathrm{B}}$ in resonance with the molecular transition $|a\rangle \rightarrow|b\rangle$. The molecules excited to the state $|b\rangle$ decay to the state $|r\rangle$ with quantum yield $\Phi$. The spectral holes are probed with a light beam of frequency $\omega$ in resonance with a transition $|a\rangle \rightarrow|c\rangle$. A possible resonance of the probe beam with a transition $|r\rangle \rightarrow|s\rangle$ of the photoproduct will also be considered.

A homogeneous subensemble within the inhomogeneous distribution is characterized by two parameters. The first is the orientation of the molecules given as the set of Euler angles $\Omega=\{\alpha, \beta, \gamma\}$ that rotate the laboratory coordinate frame into a molecular reference frame (see fig. 3 ). The second is the frequency shift $x=\omega_{b a}-\omega_{b a}^{0}$, where $\omega_{b a}=\left(E_{b}-E_{a}\right) / \hbar$ is the transition frequency for the molecular transition $|a\rangle \rightarrow|b\rangle$, and $\omega_{b a}^{0}$ is the mean value of $\omega_{b a}$ averaged over the inhomogeneous ensemble. We assume that the inhomogeneous distributions of the transitions $|a\rangle \rightarrow|b\rangle$ and $|a\rangle \rightarrow|c\rangle$ are strictly correlated, i.e. $\omega_{c a}=\omega_{c a}^{0}+x$ is determined by the same frequency shift. The transition frequency $\omega_{s r}$ of the

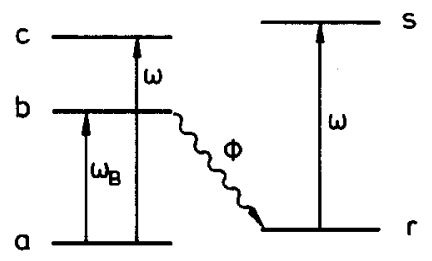

a

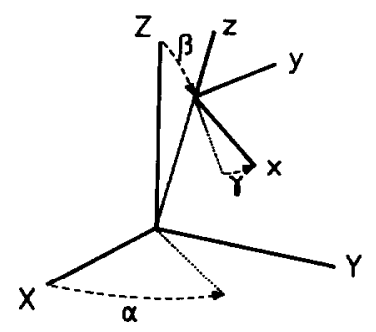

b

Fig. 3. (a) Energy level diagram used to model photochemical hole-burning. (b) Definition of Euler angles $\Omega=\{\alpha, \beta, \gamma\}$ measuring the orientation of the molecular frame $x y z$ in the laboratory frame $X Y Z$. 
photoproduct will, however, in general not be correlated with $\omega_{b a}$.

The state of the whole ensemble is then characterized by a distribution function $N_{a}(\Omega, x)$ giving the number density of molecules in the state $|a\rangle$ with orientation $\Omega$ and spectral position $x$. A similar distribution function $N_{r}(\Omega, x)$ can be defined for the photoproduct. We assume that before hole-burning no photoproduct is present, and that the initial distribution function is isotropic,

$N_{a}^{0}(\Omega, x)=N g_{0}(x) / 8 \pi^{2}$,

$N_{r}^{0}(\Omega, x)=0$.

The total density of guest molecules is $N$, and $g_{0}(x)$ is a lineshape function normalized to unity. If the molecules do not rotate or alter their spectral position $x$ within the ensemble, we have for all times

$N_{a}(\Omega, x)+N_{r}(\Omega, x)=N g_{0}(x) / 8 \pi^{2}$.

Hole-burning with light of polarization $e_{\mathrm{B}}$ and photon flux $F$ will transfer molecules to the photoproduct state with a rate

$$
\begin{aligned}
& \dot{N}_{a}(\Omega, x)=-N_{a}(\Omega, x) \Phi F \sigma_{a b}\left(\omega_{\mathrm{B}}-x\right) \\
& \quad \times\left|\boldsymbol{e}_{\mathrm{B}} \cdot \boldsymbol{e}_{a b}\right|^{2},
\end{aligned}
$$

where $e_{a b}$ is a unit vector pointing into the direction of the molecular transition dipole $\mu_{a b}$, and $\sigma_{a b}(\omega)$ is the cross section for absorption of light with frequency $\omega$ by the molecular transition $|a\rangle \rightarrow|b\rangle$ of the subensemble in the center of the inhomogeneous distribution. The population remaining in state $|a\rangle$ after time $t$ is

$$
\begin{aligned}
& N_{a}(\Omega, x)=N_{a}^{0}(\Omega, x) \\
& \quad \times \exp \left[-\Phi \bar{F} \sigma_{a b}\left(\omega_{\mathrm{B}}-x\right)\left|e_{\mathrm{B}} \cdot e_{a b}\right|^{2}\right],
\end{aligned}
$$

where $\bar{F}$ is the average photon flux.

Fig. 4a shows the spectral distribution function $N_{a}(0, x)$ for the fixed value $\Omega=0$, simulated with a Gaussian lineshape for $g_{0}(x)$ and a narrow Lorentzian lineshape for $\sigma_{a b}(\omega)$. The curves labelled 0 and 1 give the distribution function before and after holeburning, respectively. Population in a narrow spectral range around $x_{\mathrm{B}}=\omega_{\mathrm{B}}-\omega_{b a}^{0}$ is removed.

Fig. $4 \mathrm{~b}$ shows the orientational distribution function $N_{a}\left(\Omega, x_{\mathrm{B}}\right)$. With $e_{\mathrm{B}}$ chosen along the laboratory $Z$ axis and $e_{a b}$ along the molecular $z$ axis the orienta-
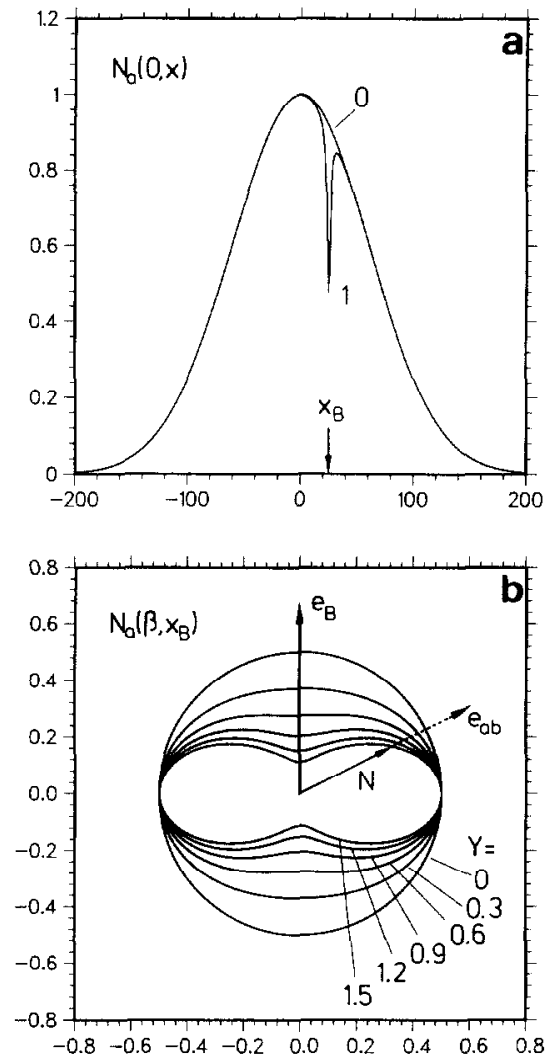

Fig. 4. (a) Spectral inhomogeneous distribution function; 0: before hole-burning, 1: after hole-burning. (b) Orientational distribution function. The length of the vector $N(\beta)$ gives the density of molecules in the ground state $|a\rangle$ whose transition dipole moment direction $\boldsymbol{e}_{a b}$ forms an angle $\beta$ with the polarization vector $e_{\mathrm{B}}$ of the hole-burning light beam. The curves are labelled with the parameter $Y=\Phi \bar{F} t \sigma_{a b}(0)$ indicating hole-burning fluence increasing in equidistant steps.

tional distribution function depends only on the Euler angle $\beta$. The length of the vector from the center of the plot to the curve gives the number density of molecules with the transition dipole pointing in the corresponding direction. Six curves have been calculated for increasing values of the parameter $Y=\Phi \bar{F} t \sigma_{a b}(0)$. The orientationally integrated photochemical conversion for the resonantly excited subensemble,

$$
\int \frac{\mathrm{d} \Omega}{8 \pi^{2}}\left[1-\exp \left(-Y \cos ^{2} \beta\right)\right]=\frac{1}{2} \sqrt{\frac{\pi}{Y}} \operatorname{erfc}(\sqrt{Y})
$$


for these six curves is $0 \%, 9 \%, 17 \%, 23 \%, 29 \%$, and $34 \%$. With increasing fluence $\bar{F} t$ the orientation distribution becomes increasingly anisotropic.

After spectral hole-burning the material is birefringent with the optical axis being $e_{\mathrm{B}}$. The response of such an anisotropic ensemble to a probe beam with input polarization $e_{\mathrm{P}}$ is schematically shown in fig. 5 . The components $E_{\|}$and $E_{\perp}$ will propagate as polarization eigenstates, but experience different attenuation and phase shifts. If the molecular transition $|a\rangle \rightarrow|c\rangle$ is polarized parallel to the transition $|a\rangle \rightarrow|b\rangle$, the distribution function $N_{a}(\beta)$ will also give the orientational distribution of the transition dipole $\boldsymbol{\mu}_{a c}$, and $\boldsymbol{E}_{\perp}$ will experience the larger attenuation as shown in fig. 5 . The probe field $E_{\text {out }}$ at the exit of the sample will have a polarization rotated towards $e_{B}$ and, in general, with an elliptical component due to the different phase shifts in $E_{\|}$and $E_{\perp}$. An analyzing polarizer oriented along $e_{\mathrm{A}}$ will transmit a field component $E_{\mathrm{A}}$ which is finally measured by a detector.

When the analyzer and polarizer are perfectly crossed $\left(e_{\mathrm{A}} \cdot e_{\mathrm{P}}=0\right)$, a signal wave $\boldsymbol{E}_{\mathrm{A}}$ is only transmitted when the frequency $\omega$ is close to a spectral

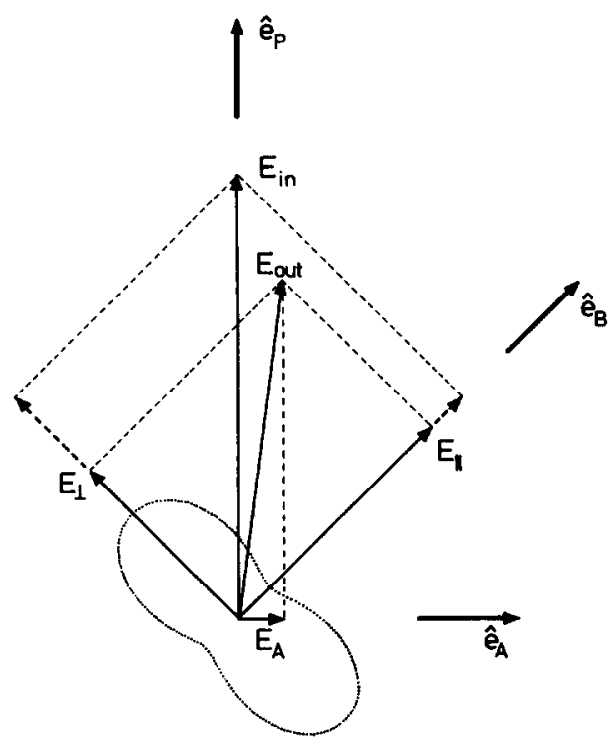

Fig. 5. Vector diagram describing the generation of the signal $\boldsymbol{E}_{\mathrm{A}}$ in polarization spectroscopy of spectral holes. The dotted line indicates the orientational distribution function. For discussion see text. hole, since the sample is isotropic for all other frequencies.

The evolution of the probe field during its propagation through the sample along a linear path $l$ is described by the one-dimensional Maxwell equation,

$\left(-\frac{\partial^{2}}{\partial l^{2}}+\frac{1}{c^{2}}(1+4 \pi \chi) \frac{\partial^{2}}{\partial t^{2}}\right) E(l, t)=0$,

where $\chi$ is the linear susceptibility tensor of the sample. Before hole-burning the sample is isotropic, the susceptibility can be treated as a scalar $\left(\chi=\chi_{0} 1\right)$, and the solution of eq. (6) is of the form

$E_{0}(l, t)=e_{\mathrm{P}} \mathscr{E}_{0} \exp \left[\mathrm{i}\left(\omega t-K_{0} l\right)\right]$,

$K_{0}=\frac{\omega}{c} \sqrt{1+4 \pi \chi_{0}}=k_{0}-\mathrm{i} a_{0}$.

The imaginary part $a_{0}$ of the complex wavevector $K_{0}$ results from the imaginary part of $\chi_{0}$ and accounts for the attenuation of the signal. The intensity at the exit of a sample with length $L$ is

$$
\begin{aligned}
I_{\mathrm{PP}} & =\frac{n c}{8 \pi} \mathscr{E}_{0} \mathscr{E}_{0}^{*} \exp \left(-2 a_{0} L\right) \\
& =I_{0} \exp \left(-2 a_{0} L\right),
\end{aligned}
$$

where $I_{0}$ is the incident intensity. Hole-burning will alter the susceptibility by

$\Delta \chi=\iint \mathrm{d} \Omega \mathrm{d} x \Delta N_{a}(\Omega, x) \boldsymbol{a}_{a c}(\Omega, \omega-x)$,

where $\boldsymbol{\alpha}_{a c}$ is the contribution of the transition $|a\rangle \rightarrow|c\rangle$ to the molecular polarizability. A perturbative treatment of Maxwell's equation with the ansatz

$E(l, t)=\left[e_{\mathrm{P}} \mathscr{E}_{0}+\Delta E(l)\right] \exp \left[\mathrm{i}\left(\omega t-K_{0} l\right)\right]$

yields for the slowly varying envelope $\Delta E(l)$

$\Delta E(l)=-\mathscr{E}_{0} \frac{2 \pi i \omega^{2} L}{c^{2} K_{0}} \Delta \chi e_{\mathrm{P}}$

The intensity of the probe beam measured through an analyzing polarizer which transmits the polarization $e_{\mathrm{A}}$ is 


$$
\begin{aligned}
I_{\mathrm{AP}} & =\frac{n c}{8 \pi}\left|e_{\mathrm{A}} \cdot E\right|^{2} \\
& =\left|e_{\mathrm{A}} \cdot e_{\mathrm{P}}-\frac{2 \pi \mathrm{i} \omega L}{c n} e_{\mathrm{A}} \Delta \chi e_{\mathrm{P}}\right|^{2} \\
& \times I_{0} \exp \left(-2 a_{0} L\right) .
\end{aligned}
$$

If the change in optical density of the sample due to spectral hole-burning is small, the intensity of the beam measured with the analyzer set parallel to the polarizer is still given by eq. (9). In this case we can define a normalized polarization spectrum as the ratio $S(\omega)=I_{\mathrm{AP}} / I_{\mathrm{PP}}$ in which the contribution from the absorption spectrum of the sample is eliminated.

The lineshape of the polarization spectrum is determined by the term $e_{\mathrm{A}} \Delta x e_{\mathrm{P}}$ in eq. (13). For small photochemical conversion it can be easily calculated. In this case eq. (4) can be expanded and truncated after the linear term

$$
\begin{aligned}
& \Delta N_{a}(\Omega, x)=-N_{a}^{0}(\Omega, x) \Phi \bar{F} t \sigma_{a b}\left(\omega_{\mathrm{B}}-x\right) \\
& \quad \times\left|\boldsymbol{e}_{\mathrm{B}} \cdot \boldsymbol{e}_{a b}\right|^{2} .
\end{aligned}
$$

Inserting this into eq. (10) leads to

$$
e_{\mathrm{A}} \Delta \chi e_{\mathrm{P}}=-\Phi \bar{F} t G H\left(\omega, \omega_{\mathrm{B}}\right),
$$

with

$$
\begin{aligned}
& G=\int \frac{\mathrm{d} \Omega}{8 \pi^{2}}\left(e_{\mathrm{B}} \cdot e_{a b}\right)\left(e_{\mathrm{B}} \cdot e_{a b}\right)\left(e_{\mathrm{A}} \cdot e_{a c}\right) \\
& \quad \times\left(e_{\mathrm{P}} \cdot e_{a c}\right), \\
& H\left(\omega, \omega_{\mathrm{B}}\right)=N \int \mathrm{d} x g_{0}(x) \sigma_{a b}\left(\omega_{\mathrm{B}}-x\right) \\
& \quad \times \alpha_{a c}(\omega-x) .
\end{aligned}
$$

The orientational average $G$ can be calculated [66] with the result

$$
\begin{aligned}
G= & \frac{1}{5}\left[\left(e_{\mathrm{A}} \cdot e_{\mathrm{B}}\right)\left(e_{\mathrm{P}} \cdot e_{\mathrm{B}}\right)-\frac{1}{3}\left(e_{\mathrm{A}} \cdot e_{\mathrm{P}}\right)\right] \\
& \times\left(\left|e_{a b} \cdot \boldsymbol{e}_{a c}\right|^{2}-\frac{1}{3}\right)+\frac{1}{9}\left(e_{\mathrm{A}} \cdot e_{\mathrm{P}}\right) .
\end{aligned}
$$

It will be discussed in section 5.2. To calculate the lineshape function $H\left(\omega, \omega_{\mathrm{B}}\right)$ reasonable assumptions for the functions $g_{0}(x), \sigma_{a b}(\omega)$ and $\alpha_{a c}(\omega)$ have to be made. The inhomogeneous distribution function $g_{0}(x)$ can in many cases be approximated by a Gaussian. If the width of $g_{0}(x)$ is much larger than that of $\sigma_{a b}(\omega)$, so that $g_{0}(x)$ will be almost constant over the whole spectral range in which $\sigma_{a b}(\omega)$ is sig- nificantly different from zero, this constant can be taken outside the integral,

$$
\begin{aligned}
& H\left(\omega, \omega_{\mathrm{B}}\right)=N g_{0}\left(\omega_{\mathrm{B}}-\omega_{b a}^{0}\right) \\
& \quad \times \int \mathrm{d} x \sigma_{a b}\left(\omega_{\mathrm{B}}-x\right) \alpha_{a c}(\omega-x) .
\end{aligned}
$$

The remaining integral is the convolution of $\sigma_{a b}(\omega)$ with $\alpha_{a c}(\omega)$. The zero-phonon contributions to $\sigma_{a b}(\omega)$ and $\alpha_{a c}(\omega)$ are

$\sigma_{a b}^{z}(\omega)=f_{a b} \frac{4 \pi \omega \mu_{a b}^{2}}{c n \hbar} \frac{\Gamma_{a b}}{\left(\omega-\omega_{b a}^{0}\right)^{2}+\Gamma_{a b}^{2}}$,

$\alpha_{a c}^{z}(\omega)=f_{a c} \frac{-\mu_{a c}^{2} / \hbar}{\omega-\omega_{c a}^{0}-\mathrm{i} \Gamma_{a c}}$,

where $f_{a b}$ and $f_{a c}$ are the Debye-Waller factors. The result of the convolution is

$$
\begin{aligned}
& H\left(\omega, \omega_{\mathrm{B}}\right)=N g_{0}\left(\omega_{\mathrm{B}}-\omega_{b a}^{0}\right) f_{a b} f_{a c} \\
& \quad \times \frac{4 \pi^{2} \omega_{\mathrm{B}} \mu_{a b}^{2} \mu_{a c}^{2}}{c n \hbar^{2}} \\
& \times \frac{-1}{\omega-\omega_{\mathrm{B}}-\omega_{c b}^{0}-\mathrm{i}\left(\Gamma_{u b}+\Gamma_{a c}\right)} .
\end{aligned}
$$

The square modulus of $H\left(\omega, \omega_{\mathrm{B}}\right)$ is a Lorentzian with peak at $\omega=\omega_{\mathrm{B}}+\omega_{c b}^{0}$ and width $W=2\left(\Gamma_{a b}+\Gamma_{a c}\right)$. This is the smallest possible width of a spectral satellite hole and it will occur in the case of perfect correlation of the inhomogeneous distributions. With less perfect correlation eq. (22) must be convoluted with the inhomogeneous distribution function of the vibrational frequency $\omega_{c b}$. Then the width will increase and the lineshape will in general no longer be Lorentzian. If several states $|c\rangle$ come into resonance with the probe beam when $\omega$ is scanned, as in type-I satellite hole-burning, eq. (22) must be summed over all states $|c\rangle$. Conversely, if several states $|b\rangle$ are excited by the hole-burning process, as in type-II satellite holeburning, summation over the index $b$ is required. The final result for the lineshape function $S(\omega)$ of the normalized polarization spectrum of zero-phonon satellite holes is then

$$
\begin{aligned}
& S(\omega)=\frac{I_{\mathrm{AP}}}{I_{\mathrm{PP}}} \\
& =\left|\sum_{b c} \frac{A_{a b c}}{\omega-\omega_{\mathrm{B}}-\omega_{c b}^{0}-\mathrm{i} \Gamma_{a b c}}+\mathrm{i} e_{\mathrm{A}} \cdot e_{\mathrm{P}}\right|^{2}
\end{aligned}
$$

with 


$$
\begin{aligned}
& \Gamma_{a b c}=\Gamma_{a b}+\Gamma_{a c}, \\
& A_{a b c}=N \Phi \bar{F} t g_{0}\left(\omega_{\mathrm{B}}-\omega_{b a}^{0}\right) f_{a b} f_{a c} \\
& \times 8 \pi^{3} \omega \omega_{\mathrm{B}}\left(\frac{\mu_{a b} \mu_{a c}}{c n \hbar}\right)^{2} G .
\end{aligned}
$$

The contribution of the photoproduct to the signal is calculated when $\Delta N_{a}, a_{a c}$, and $e_{a c}$ are replaced by $\Delta N_{r}$, $\alpha_{r s}$, and $e_{r s}$ in eq. (10). Since $\Delta N_{r}=-\Delta N_{a}$ due to eq. (2), the lineshape function is

$$
\begin{aligned}
& H\left(\omega, \omega_{\mathrm{B}}\right)=N \iint \mathrm{d} x \mathrm{~d} x^{\prime} g_{0}(x) \tilde{g}_{0}\left(x^{\prime}\right) \\
& \times \sigma_{a b}\left(\omega_{\mathrm{B}}-x\right) \alpha_{r s}\left(\omega-x^{\prime}\right) .
\end{aligned}
$$

Note that the spectral position $x^{\prime}$ within the $|r\rangle \rightarrow|s\rangle$ absorption band is not correlated with the spectral position $x$ within the $|a\rangle \rightarrow|b\rangle$ absorption band. The distribution function for $x^{\prime}$ is $\tilde{g}_{0}\left(x^{\prime}\right)$. If the educt and the product of the photochemical reaction have identical chemical structure the distribution functions $g_{0}(x)$ and $\tilde{g}_{0}(x)$ should be very similar. In this case integration of eq. (26) yields

$H\left(\omega, \omega_{\mathrm{B}}\right)=-N \bar{\sigma}_{a b}\left(\omega_{\mathrm{B}}\right) \bar{\alpha}_{r s}(\omega)$,

where $\bar{\sigma}_{a b}$ and $\bar{\alpha}_{r s}$ are the inhomogeneously broadened cross section and polarizability, respectively.

\section{Experimental}

The experimental setup is schematically shown in fig. 6. The sample, a PMMA slice doped with TPP and glued onto a $\mathrm{LiF}$ window, was mounted to the cool finger of a closed-cycle refrigerator cryostat (Leybold ROK 300-10). The original heat shield of this cryostat, which is connected to the first stage of the refrigerator operating at about $50 \mathrm{~K}$, was replaced by one of our own construction containing fused silica windows of $1 \mathrm{~mm}$ thickness. They were loosely held in place by means of steel springs to avoid strain. The IR spectrum of these windows showed that they absorb more than $99 \%$ of the radiation of a black body at $300 \mathrm{~K}$. When the original heat shield without windows was used, a carbon resistor glued to the surface of the PMMA sample indicated a temperature of $31 \mathrm{~K}$ while the cool finger was at $10 \mathrm{~K}$. This indicates a considerable temperature gradient across the thickness of the sample and the silicon rubber glue sur-

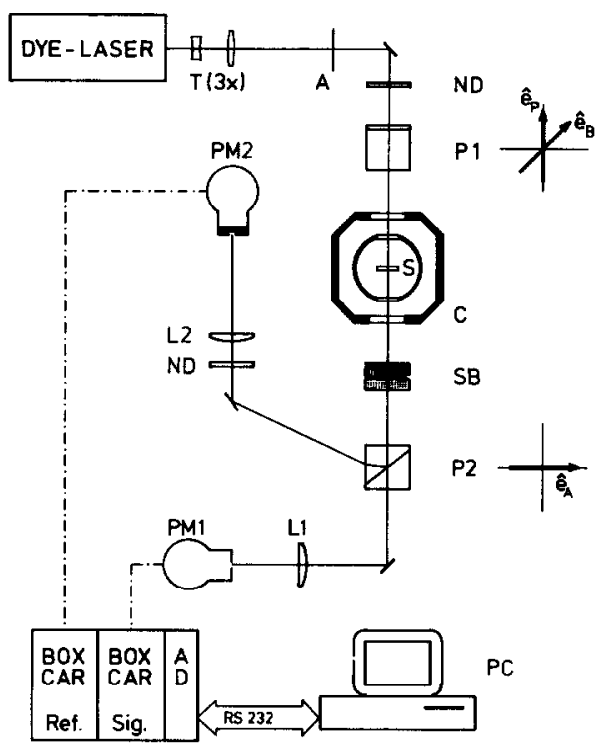

Fig. 6. Schematic diagram of the experimental setup. A; aperture, C: cryostat, L: lens, ND: neutral density filter, $P$ : polarizer, $P C$ : personal computer, PM: photomultiplier, SB: Soleil-Babinet compensator, T: teleskop, AD: analog to digital converter, $\mathrm{S}$ : sample.

rounding the carbon resistor $(\approx 2 \mathrm{~mm})$. When the new heat shield equipped with windows was used, the temperatures measured at the heat exchanger and at the outer surface of the sample were equal within experimental error ( 9.6 and $9.3 \mathrm{~K}$, respectively).

The light of a pulsed dye laser (Lambda Physik FL 2001 ) pumped by a $\mathrm{XeCl}$ excimer laser (Lambda Physik EMG 101) was used for burning and probing the spectral holes. The dyes used were rhodamine 101 and rhodamine $6 \mathrm{G}$. The bandwidth of the dye laser was less than $0.2 \mathrm{~cm}^{-1}$ as measured with a double monochromator (Spex 1402). The dye laser beam was expanded by a factor of three with a telescope and the central portion selected by an aperture of about $1 \mathrm{~mm}$ diameter. A polarizer (P1) selected the electric field component perpendicular to the experimental plane $\left(e_{\mathrm{P}}\right)$ for probing the spectral holes. For hole-burning it was rotated by $45^{\circ}\left(e_{\mathrm{B}}\right)$. Behind the sample a second polarizer (P2) was set such that it transmitted the horizontally polarized field component $\left(e_{\mathrm{A}}\right)$ which contains the signal of polarization spectroscopy. This beam was focused with a lens ( $L 1$, $f=100 \mathrm{~mm})$ through a pinhole $(400 \mu \mathrm{m})$ and mea- 
sured with a photomultiplier (PM1: 1P28). The vertically polarized component of the probe beam leaves the polarizer $\mathrm{P} 2$ through the side at an angle of $72^{\circ}$ and is imaged with a lens ( $L 2, f=230 \mathrm{~mm}$ ) onto a diffuser window in front of a photomultiplier (PM2: 1P28). The sensitivity of this second photomultiplier was reduced by using the dynode 6 as anode and wiring the dynodes 7-9 together with the old anode to a potential of about -110 Volt.

This setup allows the measurement of the transmission through crossed polarizers $\left(I_{\mathrm{AP}}\right)$ and through parallel polarizers $\left(I_{\mathrm{PP}}\right)$ of the same spot of the sample with the same laser pulse. Hence all sources of error in the ratio $I_{\mathrm{AP}} / I_{\mathrm{PP}}$ due to pulse-energy fluctuations of the dye laser, sample inhomogeneities, and long time drifts, which can arise when $I_{\mathrm{AP}}$ and $I_{\mathrm{PP}}$ are measured consecutively, are efficiently eliminated.

The electrical signals from both photomultipliers were measured with boxcar integrators (Stanford Research Systems), digitized, and transferred to a personal computer which also controlled the stepping motor of the dye laser grating drive. The ratio $I_{\mathrm{AP}} / I_{\mathrm{PP}}$ was calculated for each laser pulse. Averaging of 20 events for each wavelength setting of the dye laser yielded typical standard deviations of $1-3 \%$. The fact that the sample is composed of several materials (PMMA, silicon rubber, and LiF) with different thermal expansion coefficients inevitably leads to strain birefringence when the sample is cooled. Hence with the polarizers $P 1$ and $P 2$ crossed the extinction $I_{\mathrm{AP}} / I_{\mathrm{PP}}$ before hole-burning was often larger than $10^{-3}$. With a Soleil-Babinet compensator (SB) this birefringence could be largely compensated and the extinction ratio lowered to about $10^{-5}$.

The intensity of the dye laser was varied with neutral density filters. Pulse energies for hole-burning ranged from $1 \mu \mathrm{J} /$ pulse (20 pulses) at $645 \mathrm{~nm}$ in the region of the electronic origin band of TPP up to 11 $\mu \mathrm{J} /$ pulse ( 1000 pulses) at $580 \mathrm{~nm}$ in the vibronic band. For scanning of the spectra the laser was attenuated to about $20 \mathrm{~nJ} /$ pulse.

In many cases the spectra of $I_{\mathrm{AP}}$ and $I_{\mathrm{PP}}$ showed a small modulation containing several frequencies of a few $\mathrm{cm}^{-1}$. These apparently originated from the windows in the cryostat and the neutral density filters, which acted as etalons with low finesse. This artifact was partly suppressed by polishing all windows to wedges with an angle of about 4 arc minutes.
The preparation of the PMMA samples doped with TPP is described in the following paper [67] dealing with the measurement and interpretation of the linenarrowed spectra of this compound.

\section{Results and discussion}

\subsection{Sensitivity of the detection of spectral holes}

The drastic increase in the sensitivity of the detection of spectral holes with polarization spectroscopy is shown in fig. 7. A series of 25 equidistant spectral holes has been burned into the inhomogeneously broadened absorption band of the electronic origin of TPP in PMMA, each with a fluence of about 1.1 $\mu \mathrm{J} / \mathrm{cm}^{2}$. Subsequently the spectrum of the sample was scanned with the polarizer P1 set perpendicular to the analyzer P2 (see fig. 6). The signal $I_{\mathrm{PP}}$ rejected by the

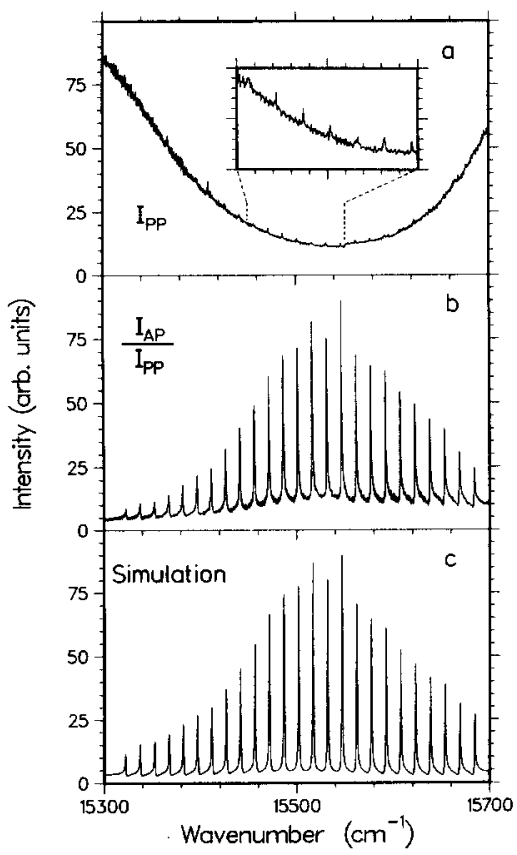

Fig. 7. Sensitivity of detection of resonant spectral holes with polarization spectroscopy. A series of equidistant holes has been burned into the inhomogeneous electronic origin band of TPP in PMMA. (a) Transmission signal $I_{\mathrm{PP}}$ through parallel polarizers. (b) Transmission signal $I_{\mathrm{AP}}$ through crossed polarizers normalized to $I_{\mathrm{Pp}}$. (c) Computer simulation of (b). 
analyzer constituting the ordinary transmission spectrum of the sample is shown in fig. 7a. The increase of transmission at the wavenumbers of the spectral holes is hardly larger than the noise (see insert with expanded scale), and the spectrum is useless for a measurement of linewidths. The polarization spectrum, i.e. the averaged ratio $I_{\mathrm{AP}} / I_{\mathrm{PP}}$ from the same scan, is shown in fig. $7 \mathrm{~b}$. In this spectrum all lines are well above the noise level and the widths can easily be determined. Between the lines the intensity does not drop to zero but descends only to a finite value. This background has three sources: (1) The phonon wings belonging to the sharp zero-phonon lines are broader than the separation of the holes and overlap. (2) At each hole-burning wavenumber molecules are excited into their phonon wing, producing additional broad overlapping holes. (3) The photoproduct has an anisotropic orientational distribution and contributes to the polarization signal. Since the transition frequencies of the product are not correlated with those of the educt, the corresponding lineshape follows the inhomogeneously broadened absorption band.

That the background in fig. $7 \mathrm{~b}$ is due to a broad resonance and not simply a constant can be shown through comparison with a computer simulation in fig. 7c. For this simulation a set of 25 complex Lorentzians with $\Gamma=0.5 \mathrm{~cm}^{-1}$ was used whose amplitude factors were taken as the square roots of the experimental intensities. This yielded a spectrum of equidistant symmetric lines. For the spectrum in fig. $7 \mathrm{c}$ an additional broad line was added with $\Gamma=125$ $\mathrm{cm}^{-1}$ and a positive amplitude. As a result of interference with this broad underlying band the sharp lines become increasingly unsymmetric with increasing distance from the center of the inhomogeneous distribution. The lines are steeper on the side that points to the center of the inhomogeneous distribution. The same behavior is seen in the experimental spectrum. A simulation with a broad underlying band with negative amplitude factor yields a completely different picture. In this case the asymmetry is reversed, and the intensity of the central lines is strongly reduced.

The phonon wings will in a first approximation have the same polarization as the corresponding zerophonon transitions. Their superposition for all the holes shown in fig. 7 will thus yield a broad complex lineshape function with a positive amplitude factor. The photoproduct produced through hole-burning of TPP is the same molecule but effectively rotated by $\pi / 2$. When the electronic origin transition of these molecules is probed, the transition dipole moment is perpendicular to its orientation during hole-burning. Thus the orientational factor $G$ will be negative (see section 5.2), but the change in population has also the opposite sign of that for the spectral holes. Hence the resulting complex lineshape function should have the width of the inhomogeneous distribution and a positive amplitude factor.

So far we have discussed resonant spectral holes whose wavenumber positions were known a priori through the preset wavenumbers for hole-burning. In the case of satellite holes the positions are not known a priori and it will be even harder to discover them in the ordinary transmission spectrum. Fig. 8 shows an example of type-II satellite holes produced by holeburning at $\nu_{B}=16281 \mathrm{~cm}^{-1}$ into the rising edge of the broad vibronic absorption band of TPP at $590 \mathrm{~nm}$ belonging to the first electronic transition. The transmitted signal through parallel polarizers $\left(I_{\mathrm{PP}}\right)$ and the normalized polarization spectrum $I_{\mathrm{AP}} / I_{\mathrm{PP}}$ of a scan over the electronic origin band are shown. The polarization spectrum displays five sharp and strong lines

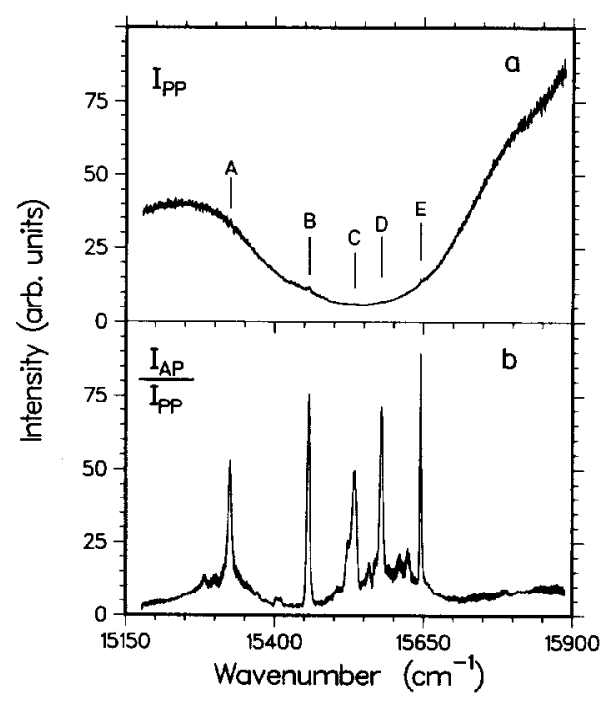

Fig. 8. Detection of type-II satellite holes in TPP following holeburning at $\nu_{\mathrm{B}}=16281 \mathrm{~cm}^{-1}$. (a) Transmission signal through parallel polarizers. (b) Normalized polarization spectrum. 
besides several weaker features. In the ordinary transmission spectrum, however, even the strong lines are hardly visible as small inflections (labelled A-E) and hardly distinguishable from noise without a foreknowledge of the line positions from the polarization spectrum.

\subsection{Positive and negative lines}

The signal measured in polarization spectroscopy is a small light field component generated by the dichroism of the sample with a polarization perpendicular to that of the incident probe wave. When the analyzing polarizer is set to block the probe wave $\left(e_{\mathrm{A}} \cdot e_{\mathrm{P}}=0\right)$, the intensity of this small component is measured. However, when the polarizer and analyzer are not set perfectly perpendicular to each other, a small fraction of transmitted probe light field will interfere with the signal light field and the resulting lineshape of the spectral holes can provide additional information.

The line-shape function $S(\omega)$ of the spectral satellite hole has been given in section 3 . For the present discussion it is written in the abbreviated form,

$S(\omega)=\left|\frac{A}{\omega-\omega_{\mathrm{H}}-\mathrm{i} \Gamma}+\mathrm{i} B\right|^{2}$,

where $\omega_{\mathrm{H}}$ is the center frequency of the spectral hole, $I$ its half-width at half maximum, and $A$ its amplitude. The term $B$ is proportional to $e_{A} \cdot e_{\mathrm{P}}$ and accounts for the transmission of the probe light when the polarizer and analyzer are not perfectly crossed.

The sign of the amplitude $A$ is the sign of the orientational average $G$ given in eq. (18). When the detuning of the polarizer and analyzer from perfect crossing is small, the factor $e_{\mathrm{A}} \cdot e_{\mathrm{P}}$ in eq. (18) can be neglected leading to

$G=(1 / 10) \sin \left(2 \phi_{\mathrm{AB}}\right)\left(\cos ^{2} \theta-1 / 3\right)$,

where $\phi_{\mathrm{AB}}$ is the angle between $e_{\mathrm{A}}$ and $e_{\mathrm{B}}$, and $\theta$ is the angle between the molecular transition dipole moments. In deriving eq. (29) we have assumed that the three polarization vectors $e_{\mathrm{A}}, \boldsymbol{e}_{\mathrm{B}}$, and $\boldsymbol{e}_{\mathrm{P}}$ lie in the same plane, and hence $\phi_{\mathrm{BP}}=\pi / 2-\phi_{\mathrm{AB}}$.

When the analyzer and polarizer are perfectly crossed, the background term $B$ vanishes and the lineshape is a Lorentzian with height $(A / \Gamma)^{2}$ and width $2 \Gamma$,
$S(\omega)=\frac{A^{2}}{\left(\omega-\omega_{\mathrm{H}}\right)^{2}+\Gamma^{2}}$.

When the polarizers are detuned from perfect crossing, a constant value is added to the imaginary part of the complex Lorentzian. The result

$S(\omega)=\frac{A^{2}+2 A B \Gamma}{\left(\omega-\omega_{\mathrm{H}}\right)^{2}+\Gamma^{2}}+B^{2}$

is a Lorentzian line superimposed on a constant background. However, whereas with $B=0$ the height of the Lorentzian is always positive, with $B \neq 0$ it can be negative as well. The conditions for this to occur are

$A B<0$ and $|A|<2|B| \Gamma$.

This means that $A$ and $B$ must have different sign and that $|B|$ must be larger than $|A| / 2 \Gamma$. Since the size and the sign of $B$ can be freely varied by detuning the polarizer or the analyzer from perfect crossing, both conditions can always be fulfilled. A negative or positive sign of $B$ is produced by increasing or decreasing the angle between $e_{\mathrm{A}}$ and $e_{\mathrm{P}}$ from $\pi / 2$. Detuning the polarizer such that the signal off resonance $\left(S=B^{2}\right)$ is larger than $1 / 4$ of the on-resonance signal with crossed polarizers $\left(S=A^{2} / \Gamma^{2}\right)$ will satisfy the second condition.

These properties of the lineshape can be used to measure the sign of $A$. If the inverted Lorentzian appears with the angle between $e_{\mathrm{A}}$ and $e_{\mathrm{P}}$ smaller than $\pi / 2$, the amplitude $A$ is negative. When the frequency of the probe light beam is set to the peak of the hole $\left(\omega=\omega_{\mathbf{H}}\right)$, detuning of the polarizer will yield a minimum in the signal for $B=-A / \Gamma$. There are two factors in eq. (29) that can cause $A$ to become negative. The first is $\sin \left(2 \phi_{\mathrm{AB}}\right)$, which is negative if $\phi_{A B}>\pi / 2$. This factor can be freely chosen by the experimenter through the choice of the polarizer setup. The second factor, $\cos ^{2} \theta-1 / 3$, depends on the angle between the two molecular transition dipole moments, and will be negative if $\theta>54.7^{\circ}$. In the following we will experimentally verify both cases.

\subsubsection{Case I: the sign of $\sin \left(2 \phi_{A B}\right)$}

When a resonant spectral hole is observed, the same transition dipole moment is involved in the holeburning and the detection step, and hence $\theta=0$. In this case the sign of $A$ is uniquely defined through the 
sign of $\sin \left(2 \phi_{\mathrm{AB}}\right)$. Fig. 9 shows an experimental demonstration of this effect (left) compared to computer simulations (right). Four spectral holes have been burned into the inhomogeneous absorption band of the electronic origin of TPP in PMMA at about $10 \mathrm{~K}$. For two of the holes the angle $\phi_{A B}$ was $\pi / 4$, i.e. $e_{B}$ was set exactly halfway in between $e_{\mathrm{A}}$ and $e_{\mathrm{P}}$. For the other two holes, $e_{\mathrm{B}}$ was rotated by $\pi / 2$. Both polarizer settings maximize the absolute value of $\left(e_{\mathrm{A}} \cdot \boldsymbol{e}_{\mathrm{B}}\right)\left(\boldsymbol{e}_{\mathrm{P}} \cdot \boldsymbol{e}_{\mathrm{B}}\right)$, and hence with $e_{\mathrm{A}}$ and $e_{\mathrm{P}}$ perfectly crossed a symmetric spectrum with four positive lines on a very low background is observed (fig. 9a). The hole-burning fluence for the two central holes was intentionally larger than that for the outer holes. When $e_{\mathrm{P}}$ is turned about $1^{\circ}$ towards $e_{\mathrm{A}}$, a positive background is added to the signal field (fig. $9 \mathrm{~b}$ ). The size of this back-

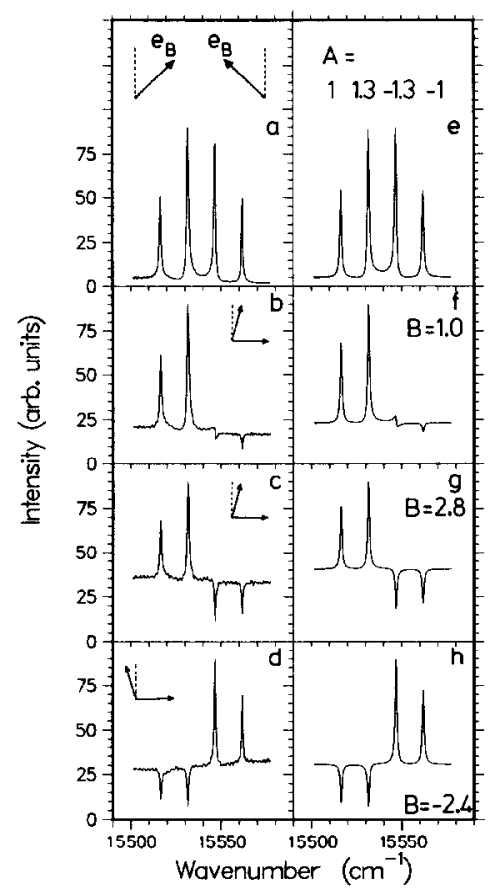

Fig. 9. Measurement of the sign of the amplitude factor of spectral holes in polarization spectroscopy. Left-hand side: experimental spectra. Four holes have been burned with the polarization direction $e_{\mathrm{B}}$ indicated on top, resulting in two holes with positive amplitude at lower wavenumbers, and two with negative sign at higher wavenumbers. (a) Polarizers $e_{\mathrm{A}}$ and $e_{\mathrm{P}}$ perfectly crossed, (b) small positive background through slight detuning of $e_{\mathrm{P}}$ towards $e_{\mathrm{A}}$, (c) large positive background, (d) negative background. Right-hand side: computer simulations. All curves are scaled to the same maximum value. ground is just sufficient to cancel the stronger one of the two lines with negative amplitude factor $A$, whereas the second is already overcompensated and appears as a small negative line. With even stronger positive detuning of $e_{\mathrm{P}}\left(\approx 2^{\circ}\right)$ both lines with negative amplitude $A$ appear as inverted lines (fig. 9c). The reversed behavior is observed when $e_{\mathrm{P}}$ is detuned away from $e_{\mathrm{A}}$, i.e. when a background field with negative sign is mixed with the signal. In this case the lines with positive amplitudes $A$ are inverted (fig. 9d).

A somewhat simplified picture of this process is obtained by the following consideration (see also fig. 5): After a spectral hole has been burned, a probe field at the same frequency experiences least absorption when it is polarized along $\boldsymbol{e}_{\mathrm{B}}$. Hence any probe field polarization will be rotated towards $e_{\mathrm{B}}$ during passage of the probe beam through the sample. When $e_{\mathrm{B}}$ is within the smaller angle formed by $e_{\mathrm{A}}$ and $e_{\mathrm{P}}$, rotation of the probe field polarization towards $e_{\mathrm{B}}$ during the scan will produce a field component $\boldsymbol{E}_{\mathrm{A}}$ pointing into the positive $e_{\mathrm{A}}$ direction. Detuning of $e_{\mathrm{P}}$ away from $e_{\mathrm{A}}$ on the other hand produces a constant field component in the negative $e_{\mathrm{A}}$ direction. In this case the rotation of the probe field polarization towards $\boldsymbol{e}_{\mathrm{B}}$ will make the polarization of the outgoing field more perpendicular to $e_{\mathrm{A}}$, and the transmitted intensity will have a minimum on resonance instead of a maximum. This picture is somewhat simplified insofar as the hole not only rotates the polarization of the probe field, but also makes it slightly elliptical, except on resonance. Hence it cannot explain the fact that for a particular detuning of the polarizer the hole spectrum will completely disappear, namely when $A=-2 B \Gamma$ in eq. (28).

The right-hand side of fig. 9 shows simulations obtained with eq. (28) containing a sum of four complex Lorentzians. The center frequencies were taken from the experimental spectrum and $\Gamma=0.6 \mathrm{~cm}^{-1}$ was assumed for all four lines. The amplitudes $A_{1}=1$, $A_{2}=1.3, A_{3}=-1.3$, and $A_{4}=-1$ were chosen for the lines from left to right. With $B=0$ this produced the curve shown in fig. 9e which is in good agreement with the corresponding experimental curve. For the curves below the values of $B=1.0,2.8$, and -2.4 were chosen by trial with the size of the polarizer detuning of the corresponding experimental curve in mind. All other parameters were left unchanged. The agree- 
ment with the experimental curves is very good. Even small details are reproduced, e.g. the small dispersion-like shape at the position of the third line in figs. $9 \mathrm{~b}$ and $9 \mathrm{f}$, which results from the interference of this line with the wings of the other lines.

In conclusion we can say that we can experimentally detect the sign of the amplitude of a spectral hole, and that the simple formula of eq. (28) with a real value of $B$ describes the experimental lineshapes very well.

At this point one could speculate about a technical use of this effect for an optical digital storage device. The sign of $A$ is a further piece of information that could be stored with each bit. Detuning of the polarizer for readout of the data could bring either set of data above the threshold for detection, whereas with perfectly crossed polarizers both sets would be available for readout.

\subsubsection{Case II: the sign of $\cos ^{2} \theta-1 / 3$}

When $e_{\mathrm{B}}$ is set within the angle formed by $e_{\mathrm{A}}$ and $e_{\mathrm{P}}$, and with $\left|e_{\mathrm{A}} \cdot \boldsymbol{e}_{\mathrm{P}}\right| \ll 1$, the factor $\left(\boldsymbol{e}_{\mathrm{A}} \cdot \boldsymbol{e}_{\mathrm{B}}\right)\left(\boldsymbol{e}_{\mathrm{B}} \cdot \boldsymbol{e}_{\mathrm{P}}\right)$ will be positive and the sign of the amplitude of a spectral hole is determined by the factor $\cos ^{2} \theta-1 / 3$ of eq. (29). A measurement of the sign of $A$ through the method outlined in the preceding section yields information on the relative orientation of transition dipoles in the molecule. A positive sign indicates $\theta<54.7^{\circ}$, a negative sign $\theta>54.7^{\circ}$. Often the symmetry of the molecule restricts $\theta$ to few values. For planar molecules belonging to point group $D_{2 h}$ the only possible values are $\theta=0$ and $\theta=\pi / 2$. A vibronic satellite hole with positive amplitude belongs then to a totally symmetric vibration $\left(a_{g}\right.$ symmetry), whereas a negative amplitude indicates $a b_{1 g}$ (in-plane) or a $\mathrm{b}_{2 \mathrm{~g}}$ (out-of-plane) vibration.

An example found in TPP doped into PMMA is shown in fig. 10. A hole has been burned at $\nu_{\mathrm{B}}=16281$ $\mathrm{cm}^{-1}$, and the satellite hole is observed in the spectral region of the electronic origin around $\nu=15460$ $\mathrm{cm}^{-1}$. When $e_{\mathrm{A}}$ and $e_{\mathrm{P}}$ are perfectly crossed, a sharp line is observed which peaks at $15459 \mathrm{~cm}^{-1}$, i.e. 822 $\mathrm{cm}^{-1}$ below $\nu_{\mathrm{B}}$ (fig. $10 \mathrm{~b}$ ). The line appears slightly unsymmetric with a width of $5.5 \mathrm{~cm}^{-1}$. In fig. $10 \mathrm{a}$ the polarizer $e_{\mathrm{P}}$ was turned away from $e_{\mathrm{A}}$ by about $2^{\circ}$ leading to a negative background $B$. Thus a line with positive amplitude should be inverted, whereas one with negative amplitude should increase in intensity.

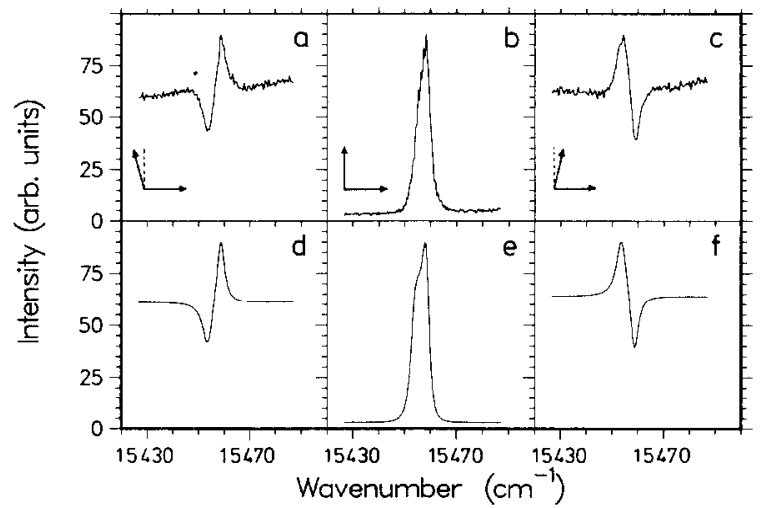

Fig. 10. Experimental determination of the sign of amplitudes of vibrational satelliteholes. (a) Negative background field added $\left(e_{\mathrm{A}} \cdot e_{\mathrm{P}}<0\right)$. (b) Crossed polarizers. (c) With positive background field, $e_{\mathrm{A}} \cdot e_{\mathrm{p}}>0$. (d) - (f) Computer simulations.

The behavior of the line in fig. 10a is, however, quite unexpected: a negative peak at $15454 \mathrm{~cm}^{-1}$ and a positive peak at $15459 \mathrm{~cm}^{-1}$ result. Detuning the polarizer towards the analyzer leads to the completely reversed picture, shown in fig. 10c. On first glance one could be tempted to assign this lineshape to a dispersion-like shape, which could indeed be generated with eq. (28) when the value of $B$ is purely imaginary. However, with the lineshapes collected in fig. 9 we have shown that detuning of the polarizer yields a purely real value of $B$. Furthermore, all other satellite holes in the vicinity of that shown in fig. 10 behave quite normally: some are inverted for positive background, the others for negative background. The conclusion then is that the spectral feature observed in fig. 10 is a pair of vibronic states with opposite sign of amplitudes. In the lower part of fig. 10 the situation has been simulated with two lines with amplitudes $A_{1}=1.08$ and $A_{2}=-1.0$, and width $\Gamma_{1}=2.4$ $\mathrm{cm}^{-1}$ and $\Gamma_{2}=1.8 \mathrm{~cm}^{-1}$. With $B=-2.2$ in fig. $10 \mathrm{~d}$, $B=0$ in fig. $10 \mathrm{e}$, and $B=2.1$ in fig. $10 \mathrm{f}$ we obtain lineshapes which agree quite well with the experimental ones. This shows that the technique of detuning of the polarizers not only yields information on the symmetry of the vibronic states, but also can increase the resolution of the method through separation of otherwise unresolved lines. 


\section{Conclusions}

(1) Spectral hole-burning can be used to obtain the line-narrowed vibrationally resolved spectrum of an electronic transition in an amorphous material in which this spectrum is normally obscured by inhomogeneous broadening.

(2) Polarization spectroscopy is a sensitive method for the detection of spectral holes. When the analyzing polarizer is perfectly crossed with the probe-beam polarizer, transmission of the probe beam is only observed at the frequencies of the spectral holes.

(3) Detuning of the polarizer from perfect crossing with the analyzer mixes a small background field of either positive or negative phase to the signal. Spectral holes with a negative (positive) amplitude factor will appear as inverted Lorentzian lines if a sufficiently strong positive (negative) background field is mixed to the signal.

(4) The sign of the amplitude factor of a spectral hole contains information about the relative orientation of transition dipoles and hence about the symmetry of the vibronic states.

(5) If the inhomogeneous distributions of the electronic origin and the vibronic transition are fully correlated, the linewidth of a vibronic satellite hole is the mean linewidth of the two corresponding resonant holes and is determined by the phase-relaxation times of the individual vibronic states.

The results obtained so far are encouraging for the development of this technique as a method to obtain line-narrowed spectra of guest molecules in amorphous environments. Several improvements should be possible:

- Use of a liquid-helium cryostat instead of the refrigerator cryostat used in the present investigation will allow us to obtain temperatures below $10 \mathrm{~K}$, which should lead to further narrowing of the lincs and a better contrast of the zero-phonon lines compared to the phonon sidebands.

- When the sample is cooled with cold helium gas or with liquid helium, no substrate and glue are necessary to establish good thermal contact. This will result in a more homogeneous temperature profile across the sample and reduce most of the strain birefringence.

- The method is not restricted to the detection of photochemically produced spectral holes. In fact, transient hole-burning employing a metastable bottleneck state can be applied to many molecules which do not react photochemically. We have already applied polarization spectroscopy to the detection of spectral holes which were transient on a nanosecond timescale. These results will be published elsewhere.

- The method could be further improved through multiplexed detection of the spectral holes with an optical multichannel detector.

\section{Acknowledgement}

The author is greatly indebted to Professor F.P. Schäfer for generous support of this work and to Dr. $T$. Jovin for the loan of the Soleil-Babinet compensator. Dr. J. Jasny made valuable suggestions concerning the experimental apparatus. Helpful discussions with Professor W. Lüttke and Dr. B. Nickel are gratefully acknowledged. This work was supported by the Deutsche Forschungsgemeinschaft through the Leibniz-Prize program and project SFB 93 (Photochemie mit Lasern ).

\section{References}

[1] R.A. McFarlane, W.R. Bennett Jr. and W.E. Lamb Jr., Appl. Phys. Letters 2 (1963) 189.

[2] A. Szöke and A. Javan, Phys. Rev. Letters 10 (1963) 521.

[3] C. Freed and A. Javan, Appl. Phys. Letters 17 (1970) 53.

[4] T.W. Hänsch, M.D. Levenson and A.L. Schawlow, Phys. Rev. Letters 26 (1971) 946.

[5] M.D. Levenson and A.L. Schawlow, Phys. Rev. A 6 (1972) 10.

[6] M.S. Sorem and A.L. Schawlow, Opt. Commun. 5 (1972) 148.

[7] T.W. Hänsch, M.H. Nayfeh, S.A. Lee, S.M. Curry and I.S. Shakin, Phys. Rev. Letters 32 (1974) 1336.

[8] F. Biraben, B. Cagnac and G. Grynberg, Phys. Rev. Letters 32 (1974) 643.

[9] T.W. Hänsch, K. Harvey, G. Meisel and A.L. Schawlow, Opt. Commun. 11 (1974) 50.

[10] M.D. Levenson and N. Bloembergen, Phys. Rev. Letters 32 (1974) 645.

[11] F. Gelbwachs, P.F. Jones and J.E. Wessel, Appl. Phys. Letters 27 (1975) 551.

[12] R.E. Smalley, D.H. Levy and L. Wharton, J. Chem. Phys. 64 (1976) 3266.

[13] D.H. Levy, L. Wharton and R.E. Smalley, Accounts Chem. Res. 10 (1977) 134.

[14] D.H. Levy, Ann. Rev. Phys. Chem. 31 (1980) 197. 
[15] A. Szabo, Phys. Rev. Letters 25 (1970) 924.

[16] A. Szabo, Phys. Rev. Letters 27 (1971) 323.

[17] D.A. Wiersma, Advan. Chem. Phys. 47 (1981) 421.

[18] R.I. Personov, E.I. Al'shits and L.A. Bykovskaya, Opt. Commun. 6 (1972) 169.

[19] L.A. Riseberg, Phys. Rev. A 7 (1973) 671.

[20] J.L. Oudar and Y.R. Shen, Phys. Rev. A 22 (1980) 1141.

[21 ] S.A.L. Druet, J.P.E. Taran and Ch.J. Borde, J. Phys. (Paris) 40 (1979) 841.

[22] S.A.L. Druet, J.P.E. Taran and Ch.J. Borde, J. Phys. (Paris) $41(1980) 183$.

[23] B. Dick and R.M. Hochstrasser, J. Chem. Phys. 78 (1983) 3398.

[24] R. Bozio, P.D. DeCola and R.M. Hochstrasser, in: TimeResolved Vibrational Spectroscopy, ed. G.H. Atkinson (Academic Press, New York, 1983) p. 335.

[25] P.L. DeCola, J.R. Andrews, R.M. Hochstrasser and H.P. Trommsdorff, J. Chem. Phys. 73 (1980) 4695.

[26] J.R. Andrews and R.M. Hochstrasser, Chem. Phys. Letters 83 (1981) 427.

[27] J.R. Andrews, R.M. Hochstrasser and H.P. Trommsdorff, Chem. Phys. 62 (1981) 87

[28] A.R. Bodgan, Y. Prior and N. Bloembergen, Opt. Letters 6 ( 1981$) 82$.

[29] N. Bloembergen, H. Lotem and R.T. Lynch Jr., Indian J. Pure Appl. Phys. 16 (1977) 151.

[30] R.M. Hochstrasser and H.P. Trommsdorff, Accounts Chem. Res. 16 (1983) 376.

[31 ] L.A. Rebane, Appl. Phys. B 29 (1982) 235.

[32] J. Friedrich and D. Haarer, Angew. Chem. 96 (1984) 96.

[33] S. Völker, R.M. Macfarlane and J.H. van der Waals, Chem. Phys. Letters 53 (1978) 8.

[ 34 ] H.P.H. Thijssen, S. Völker, M. Schmidt and H. Port, Chem. Phys. Letters 94 (1983) 537.

[35] H.P.H. Thijssen, R. van den Berg and S. Völker, Chem. Phys. Letters 103 (1983) 23.

[36] S. Völker and R.M. Macfarlane, Chem. Phys. Letters 61 (1979) 421 .

[37] A.I.M. Dicker and S. Völker, Chem. Phys. Letters 87 (1982) 481.

[38] B. Dick and B. Nickel, Chem. Phys. 110 (1986) 131.

[39] V.G. Maslow, Opt. Spectry. 50 (1981) 599.

[40] B.M. Kharlamov, L.A. Bykovskaya and R.I. Personov, Chem. Phys. Leters 50 (1977) 407.

[41] A.A. Gorokhovski and J. Kikas, Opt. Commun. 21 (1977) 272.

[42] J. Friedrich and D. Haarer, J. Chem. Phys. 79 (1983) 1612.
[43] C. Wieman and T.W. Hänsch, Phys. Rev. Letters 36 (1976) 1170.

[44] R. Teets, R. Feinberg, T.W. Hänsch and A.L. Schawlow, Phys. Rev. Letters 37 (1976) 683.

[45] R.E. Teets, F.V. Kowalski, W.T. Hill, N. Carlson and T.W. Hänsch, in: Advances in Laser Spectroscopy, Proc. Soc. Phot. Opt. Instrum. Eng., Vol. 113, San Diego, 1977, p. 80.

[46] J.J. Song, J.H. Lee and M.D. Levenson, Phys. Rev. A 17 (1978) 1439.

[47] Y. Taira and Y. Yajima, J. Phys. Soc. Japan 50 (1981) 3459.

[48] S. Saikan and J. Sei, J. Chem. Phys. 79 (1983) 4146, 4154.

[49] F. Garcia-Golding, J. Opt. Soc. Am. 73 (1983) 59.

[50] A. Marcano O., R.A. Abreu and F. Garcia-Golding, J. Phys. B 17 (1984) 2151.

[51] A. Marcano O. and F. Garcia-Golding, J. Chem. Phys. 82 (1985) 1242.

[52 ] J.H. Lee, J.J. Song, M.A.F. Scarparo and M.D. Levenson, Opt. Letters 5 (1980) 196.

[53] M.D. Levenson, R.M. Macfarlane and R.M. Shelby, Phys. Rev. B 22 (1980) 4915.

[54] M. Romagnoli, M.D. Levenson and G.C. Bjorklund, J. Opt. Soc. Am. B 1 (1984) 571.

[55] A.A. Gorokhovski, P.K. Kaarli and L.A. Rebane, J. Exp. Theoret. Phys. Letters 20 (1974) 216; Opt. Commun. 16 (1976) 282.

[56] H. de Vries and D.A. Wicrsma, Phys. Rev. Letters 36 (1976) 91.

[57] S. Völker and J.H. van der Waals, Mol. Phys. 32 (1976) 1703.

[58] S. Völker, R.M. Macfarlane, A.Z. Genack, H.P. Trommsdorff and J.H. van der Waals, J. Chem. Phys. 67 (1977) 1759

[59] B.M. Kharlamov, R.I. Personov and L.A. Bykovskaya, Opt. Commun. 12 (1974) 191.

[60] J.M. Hayes and G.J. Small, Chem. Phys. 27 (1978) 151.

[61 ] G.J. Small, in: Spectroscopy and Excitation Dynamics of Condensed Molecular Systems, eds. V.M. Agranovich and R.M. Hochstrasser (North-Holland, Amsterdam, 1983) p. 515.

[62] R.M. Shelby and R.M. Macfarlane, Chem. Phys. Letters 64 (1979) 545.

[63] A. Szabo, Phys. Rev. B 11 (1975) 4512.

[64] A.I.M. Dicker, L.W. Johnson, S. Völker and J.H. van der Waals, Chem. Phys. Letters 100 (1983) 8.

[65] B. Dick, Chem. Phys. Letters 143 (1988) 186.

[66] B. Dick, Chem. Phys. 113 (1987) 131.

[67] B. Dick, Chem. Phys. 136 (1989) 429. 